\title{
Effect of Soliton Interaction on Timing Jitter in Communication Systems
}

\author{
Armando Nolasco Pinto, Student Member, OSA, Govind P. Agrawal, Fellow, IEEE, Fellow, OSA, \\ and J. Ferreira da Rocha
}

\begin{abstract}
Timing jitter in soliton communication systems is studied, taking into account both soliton interaction and amplifier noise. Deviations from Gordon-Haus jitter for closely spaced solitons are observed. A new analytical model for the timing jitter is proposed. The model presented considers interaction in a random sequence of solitons and the effect of the amplified spontaneous emission noise added in each amplification stage. We obtain a good agreement between the new analytical model and simulation results for practical communication systems.
\end{abstract}

Index Terms - Communication systems, solitons, timing jitter.

\section{INTRODUCTION}

I $\mathrm{N}$ a long-haul soliton communication system, lumped amplifiers are used to compensate for fiber losses. The noise added to the signal by each amplifier induces an uncertainty in the soliton arrival time called jitter. Gordon and Haus showed that the statistics of the jitter due to the spontaneous emission noise added by lumped amplifiers is Gaussian, with a variance proportional to the cube of the total distance of the link [1].

Recent experiments have shown significant deviations from the Gaussian distribution [2]. The study of the physical effects leading to this deviation has been the subject of considerable amount of study. It was pointed out that soliton interaction, acoustic effects, and polarization mode dispersion can lead to deviations from the Gordon-Haus result [3], [4]. However, the soliton interaction is likely to have the dominant effect for high bit rate systems.

In this work we derive a model for the soliton arrival time statistics considering the soliton interaction and the amplified spontaneous emission noise. As full Monte Carlo simulations for arrival time calculation require huge amount of computing time, this new model can be useful in system design [5], [6]. We validate this new model with numerical simulations of practical communication systems, working at 10,20, and $40 \mathrm{~Gb} / \mathrm{s}$. The results show that soliton interaction produces a considerable change in the jitter statistics.

\section{DEVIATION From the GaUSSIAN STATISTICS (SimUlation RESUlT)}

Gordon-Haus jitter due to the spontaneous emission noise

Manuscript received May 12, 1997. This work was supported by a JNICT grant under the PRAXIS XXI program and by ACTS project ESTHER.

A. N. Pinto and J. Ferreira da Rocha are with the Department of Electronic and Telecommunications, University of Aveiro, Aveiro 3810 Portugal.

G. P. Agrawal is with the The Institute of Optics, University of Rochester, Rochester NY 14627 USA.

Publisher Item Identifier S 0733-8724(98)02586-9.
TABLE I

\begin{tabular}{l|c}
\hline Bit rate & $20 \mathrm{Gbit} / \mathrm{s}$ \\
\hline Pulse width & $10.0 \mathrm{ps}$ \\
\hline Wavelength & $1.55 \mu \mathrm{m}$ \\
\hline Total length & $3000 \mathrm{~km}$ \\
\hline Span between amplifiers & $20 \mathrm{~km}$ \\
\hline Dispersion & $0.1 \mathrm{ps} /(\mathrm{nm} . \mathrm{km})$ \\
\hline Attenuation & $0.1 \mathrm{~dB} / \mathrm{km}$ \\
\hline Spontaneous emission factor & 2.0 \\
\hline Nonlinear coefficient & $3.7 \mathrm{~W}^{-1} \cdot \mathrm{km}^{-1}$ \\
\hline
\end{tabular}

added in each amplifier is described by a Gaussian probability density function (pdf) with the standard deviation given by [1]

$$
\sigma=\left[\frac{n_{\mathrm{sp}} n_{2} D h(G-1) L^{3}}{9 T_{o} A_{\mathrm{eff}} L_{\mathrm{amp}} Q}\right]^{1 / 2}
$$

where $n_{\mathrm{sp}}$ is the spontaneous emission factor, $n_{2}$ is the nonlinear parameter of the fiber, $D$ is the first-order groupvelocity dispersion factor of the fiber, $h$ is Planck's constant, $G$ is the amplifier gain, $L$ is the total length of the link, $T_{o}$ is the soliton width, $A_{\text {eff }}$ is the effective mode area, $L_{\mathrm{amp}}$ is the amplifier spacing, and $Q$ is the power-enhancement factor [7].

In communication systems, the solitons are launched close to each other, in order to achieve high-bit rates, which leads to mutual interactions. This perturbation of the soliton propagation due to neighbor pulses significantly changes the timing jitter statistics.

To study the effect of the soliton interaction we have solved the nonlinear Schrödinger equation (NLSE) for a pseudorandom sequence of solitons. We considered an optical communication system operating at $20 \mathrm{~Gb} / \mathrm{s}$, with the parameters shown in Table I.

The separation between amplifiers was kept much less than the dispersion length, in order to avoid the dispersive waves that could become another source of timing jitter. We have solved numerically the NLSE equation for 1600 bits from a pseudo-random sequence, using 64 sample points for each bit slot for accurate jitter measurements. Fig. 1 shows the pdf of the arrival time obtained by simulation and the Gordon-Haus result.

We clearly see the deviation from the Gordon-Haus result due to the soliton interaction. The pdf of the arrival time is not Gaussian and it is broader, so we should expect a degradation of the system bit error rate due to soliton interaction. 


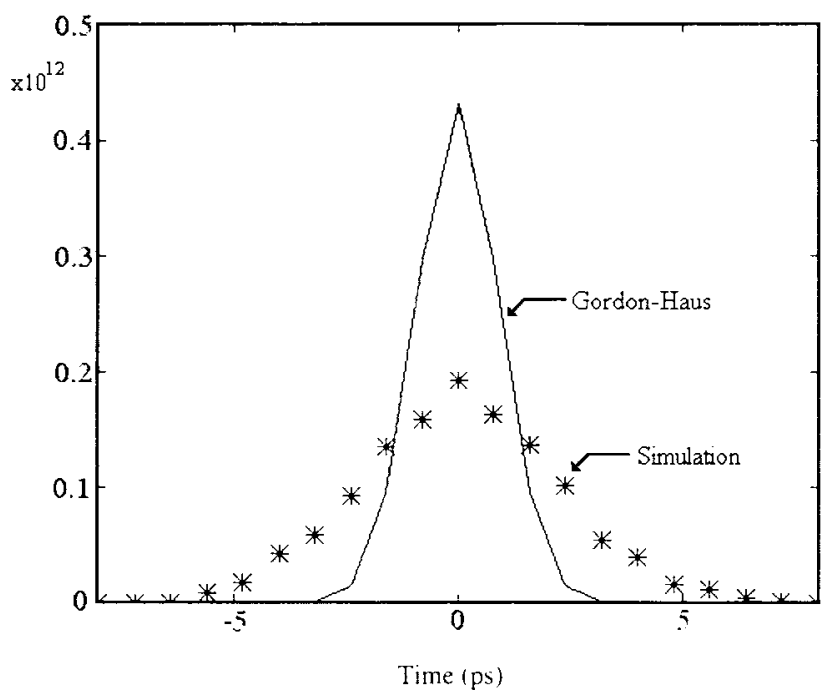

Fig. 1. Probability density function for the timing jitter of a $20 \mathrm{~Gb} / \mathrm{s}$ system. The line is the Gordon-Haus result and the starts are the simulation result.

\section{ANALyticAl Model of SOliton InTERACTION}

When we launch more than one pulse in a fiber, the presence of neighbor pulses disturbs the soliton, which changes its time position. If the neighbor solitons are in phase, this interaction is attractive and solitons move closer.

To study multiple-pulse systems, we start with the NLSE for the case of two solitons. An approximate solution for the case of two solitons based on the quasiparticle approach was presented by Karpman and Solov'ev [8]. Another approximation, was derived by Gordon directly from the exact two solitons equation [9]. The expression for a soliton pair has the general form

$$
\begin{aligned}
u(\tau, q)=\exp (i \Omega)\left\{A_{1} \operatorname{sech}\left[A_{1}(\tau-q)\right] \exp \left(i \theta_{1}\right)\right. \\
\left.+A_{2} \operatorname{sech}\left[A_{2}(\tau+q)\right] \exp \left(i \theta_{2}\right)\right\} .
\end{aligned}
$$

At each point inside the fiber the normalized separation between the solitons is $2 q$, the amplitudes of the two solitons are $A_{1}$ and $A_{2}$, and the relative phase difference is $\theta_{2}-\theta_{1}$. For simplicity of notation, we introduce $\psi=\left(\theta_{2}-\theta_{1}\right) / 2$.

It can be shown that

$$
\rho \exp (q+i \psi)=2 \cosh \left(\zeta_{\circ}+i \rho \zeta\right)
$$

where $\zeta$ is the distance of propagation, $\rho$ and $\zeta_{0}$ are constants determined by the initial separation, phase difference and its first derivative. The first and second space derivatives of (3) combine to yield the equations of motion [8], [9]

$$
\frac{\partial^{2} q}{\partial \zeta^{2}}=-4 \exp (-2 q) \cos (2 \psi)
$$

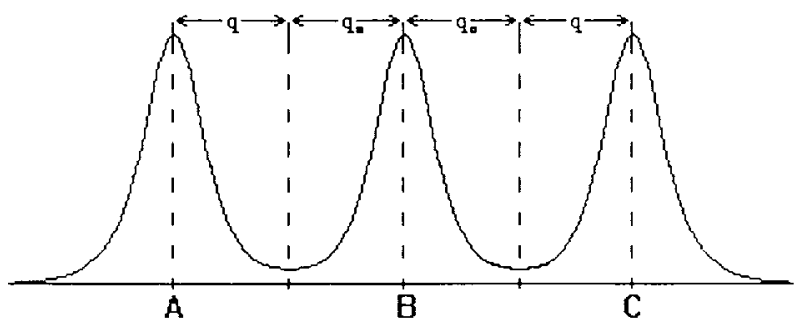

Fig. 2. In the three-soliton system in phase, the interactions of solitons A and $\mathrm{C}$ relative to $\mathrm{B}$ are of opposing signs.

$$
\frac{\partial^{2} \psi}{\partial \zeta^{2}}=4 \exp (-2 q) \sin (2 \psi)
$$

Equations (4) and (5) show that the dynamics of the soliton pair are entirely due to interaction forces that depend exponentially on their separation and sinusoidally on their relative phase. If $q_{o}$ and $\psi_{o}$ are the initial separation and phase difference respectively, we obtain the followings expressions for $q$ and $\psi$ during the propagation in (6) and (7) shown at the bottom of the page.

Since a zero phase difference between neighboring solitons leads to the worst case, we consider this case only. Choosing $\psi_{0}=0$ in (6), we obtain the following expression for the separation between the two solitons:

$$
q=q_{o}+\ln [\cos (a \zeta)]
$$

where $a=2 \exp \left(-q_{o}\right)$.

To extend the above results to the three-soliton case, we note that the force between adjacent solitons depends on their separation and relative phases. Then in a system of three solitons where the distance and relative phases between side solitons (Fig. 2, A and C) and the middle soliton B are the same, the interaction of solitons $\mathrm{A}$ and $\mathrm{C}$ relative to $\mathrm{B}$, are of opposing signs. The middle soliton is fixed because of these balancing forces.

In the two-soliton case, the separation is equal to $2 q$. However, in the three-soliton case, because the middle soliton is fixed, the separation between side solitons and the middle one is $q_{o}+q$ where $q_{o}$, is the initial separation. If we use this fact in (4) and (5), we obtain the following pair of equations for the three-soliton dynamics:

$$
\begin{aligned}
& \frac{\partial^{2} q}{\partial \zeta^{2}}=-2 a \exp (-q) \cos (2 \psi) \\
& \frac{\partial^{2} \psi}{\partial \zeta^{2}}=2 a \exp (-q) \sin (2 \psi)
\end{aligned}
$$

where $a=2 \exp \left(-q_{o}\right)$.

$$
\begin{aligned}
& q(\zeta)=q_{o}+\frac{1}{2} \ln \left[\frac{\cosh \left[4 \exp \left(-q_{o}\right) \sin \left(\psi_{o}\right) \zeta\right]+\cos \left[4 \exp \left(-q_{o}\right) \cos \left(\psi_{o}\right) \zeta\right]}{2}\right] \\
& \psi(\zeta)=\psi_{o}+\frac{1}{2 i} \ln \left[\frac{\cos \left[2 \exp \left(-q_{o}\right) \exp \left(-i \psi_{o}\right) \zeta\right]}{\cos \left[2 \exp \left(-q_{o}\right) \exp \left(i \psi_{o}\right) \zeta\right]}\right]
\end{aligned}
$$




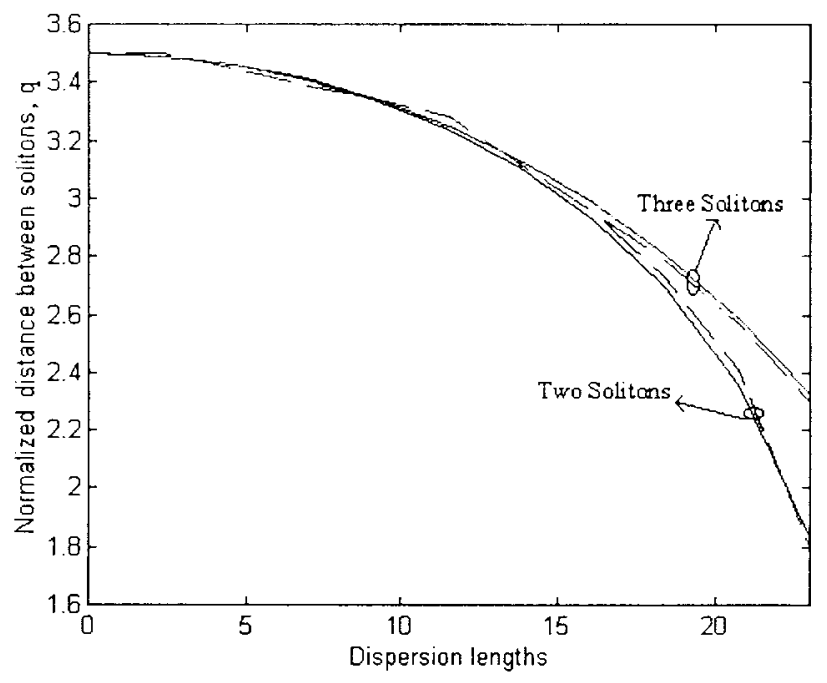

Fig. 3. Normalized distance between solitons. The solid line is the analytical solution, the dashed line is obtained by numerical solution of the NLSE.

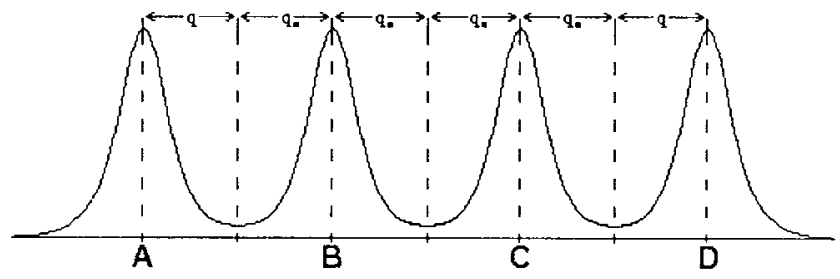

Fig. 4. In the four-soliton system the middle solitons (B and C) are practically fixed because each one is surrounded by neighbor pulses exerting opposing forces.

The solution of (9) and (10), in the case of in-phase pulses provides:

$$
q=q_{o}+\ln \left[\cos ^{2}\left(\frac{1}{\sqrt{2}} \alpha \zeta\right)\right] .
$$

Comparing (11) with the corresponding solution (8) for the two solitons case, we see that despite the square in the cosine function, in the case of three solitons, the period is $\sqrt{2}$ times the period of the two-soliton system. This means that the soliton interaction is weaker for the case of three-soliton compared to the case of two-soliton.

To test the validity of our analytical result, we performed a simulation by numerically solving the NLSE for the case of two and three-soliton. The results are shown in Fig. 3. As we can see, the numerical results are in agreement with the analytical ones.

To generalize our results, we start with the four-soliton case, in which the two middle solitons (B and C in Fig. 4) are practically fixed, because each one is surrounded by neighbor pulses exerting opposing forces. With a good degree of approximation, we can assume that the two middle solitons are fixed and the side solitons (A and D) behave in a similar way to the three-soliton system.

It is obvious that we can extend this approximation to the case of more than four solitons. In a long sequence of pulses, the only ones where the interaction forces are important are the first and the last one of the sequence. So the case of more than three solitons can be reduced to the three-soliton system.

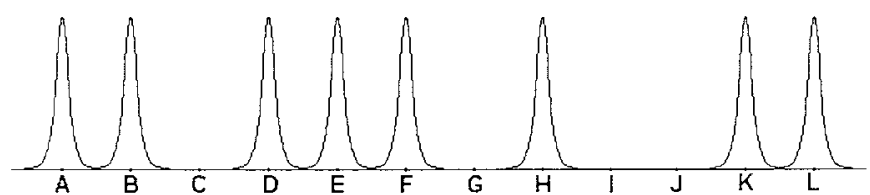

Fig. 5. To analyze the solitons interaction in a long sequence of pulses we can divide the sequence in small slices and analyze each slice.

In a lightwave communication system making use of pulsecode modulation, the pulse sequence is random, containing long sequences of pulses, but also isolated and pairs of pulses. To analyze the solitons interaction in a long sequence of pulses, we can divide the sequence in small slices and reduce each slice to one of the cases presented above.

In Fig. 5, we have a sequence of 12 bits that we can reduce to the cases of isolated pulse (H), two-soliton case (A and B, $\mathrm{K}$, and $\mathrm{L}$ ) and three-soliton system (D and $\mathrm{E}$ and $\mathrm{F}$ ).

In order to analytically determine the arrival time statistics, it is enough to consider at most four neighbor solitons because the interaction forces decrease exponentially with solitons separation. We consider a random binary sequence with $2^{5}-1$ bits that contains all possible sequences of 5 bits [10].

It can be shown that the statistics of the arrival times is represented by a bar graph, with 50\% weight for zerotime deviation, $25 \%$ corresponding to a deviation equal to the two-soliton system, and $25 \%$ with a deviation equal of the three-soliton system. Moreover, since the deviation can be positive or negative the $25 \%$ probability of two and three solitons should be split into two bars of $12.5 \%$ on the two sides. So, we should expect a jitter pdf given by

$$
\begin{aligned}
p(t)= & \frac{1}{2} \delta(t)+\frac{1}{8} \delta\left(t-t_{2}\right)+\frac{1}{8} \delta\left(t+t_{2}\right) \\
& +\frac{1}{8} \delta\left(t-t_{3}\right)+\frac{1}{8} \delta\left(t+t_{3}\right) .
\end{aligned}
$$

Values of $t_{2}$ and $t_{3}$ are given by

$$
\begin{aligned}
& t_{2}=T_{o} \ln \left[\cos \left(\frac{a}{L_{D}} z\right)\right] \\
& t_{3}=T_{o} \ln \left[\cos ^{2}\left(\frac{a}{\sqrt{2} L_{D}} z\right)\right]
\end{aligned}
$$

where $T_{o}$ is the soliton width, $L_{D}$ the dispersion length, $a=2 \exp \left[-T_{\mathrm{bit}} /\left(2 T_{o}\right)\right], T_{\mathrm{bit}}$ is the bit period and $z$ is the total length of the optical communication system.

To check the validity of our analytical result, we repeat the $20 \mathrm{~Gb} / \mathrm{s}$ system simulation, described in Section II, with an ideal amplifier $\left(n_{\mathrm{sp}}=0\right)$ and with a total length of nine dispersion lengths. From Fig. 6 we can see a good agreement between our analytical deduction and the numerical results. There are a broadening in the delta function produced by the finite numerical resolution (resolution $=0.78 \mathrm{ps}$ ), but the position of the side bars are exactly the positions predicted by our model and their relative heights match the prediction of (12).

\section{EFFECt of SPONTANEOUS-EMISSION NOISE}

In a long-haul soliton communication system, the fiber losses are compensated by the use of in line lumped amplifiers. These amplifiers restore the signal power but the amplification 


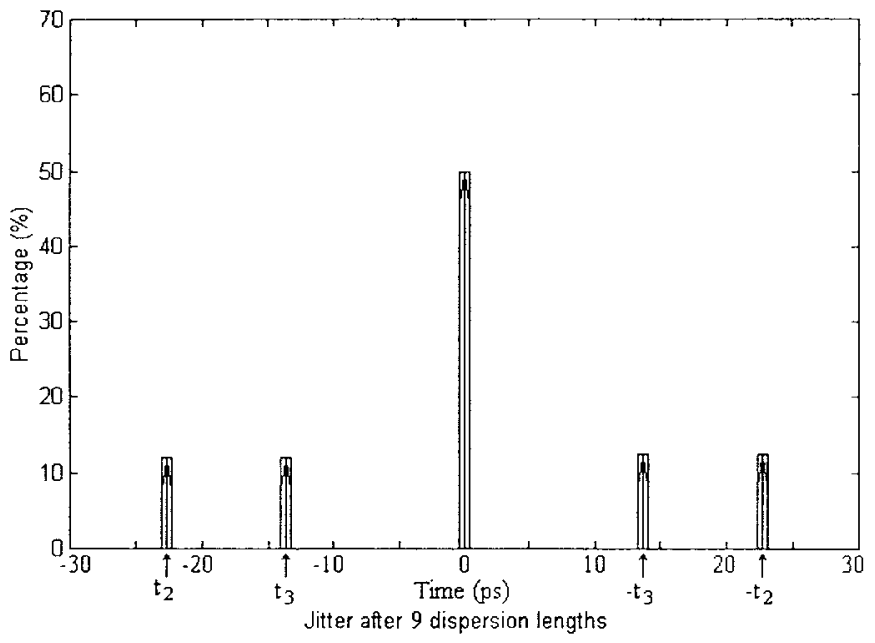

Fig. 6. Arrival time statistics of a pseudorandom sequence, after the propagation of nine dispersion lengths. The bars were obtained by numerical simulation. We used expressions (13) and (14) to determine the $t_{2}$ and $t_{3}$. The arrows inside the bars are the delta function obtained by expression (12).

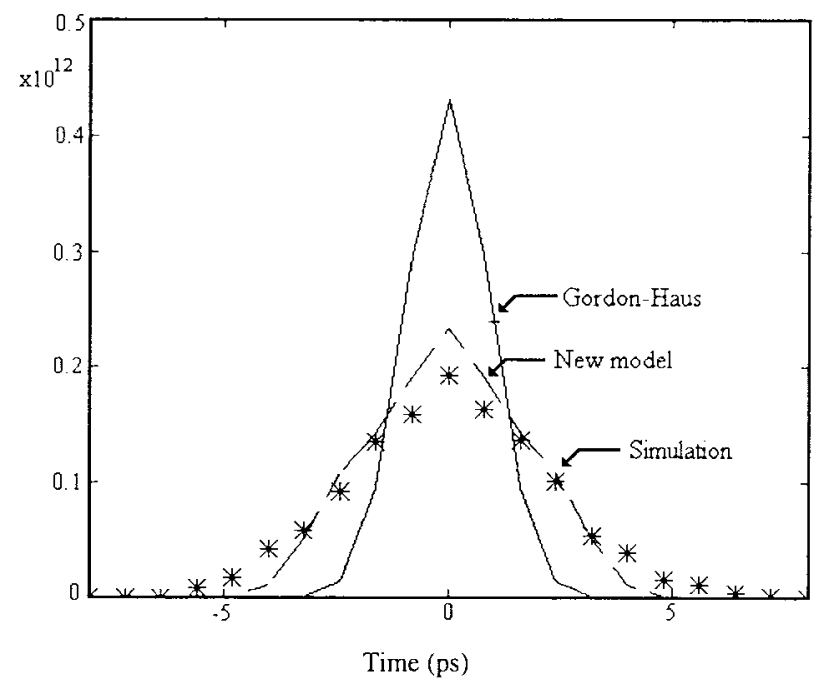

Fig. 7. Probability density function for the $20 \mathrm{~Gb} / \mathrm{s}$ system. The line is the Gordon-Haus result, the starts are the simulation result, and the dashed-line is our model.

process is accompanied by the emission of spontaneous noise [11]. The noise that is outside the bandwidth of the optical signal can be removed, using an appropriate optical filter, although it is not possible to remove in-band noise. This noise copropagates with the signal.

The copropagation of signal and noise changes the arriving time statistics presented in expression (12) and also degrades the signal noise ratio. Expression (12) is not valid any more, due to the Gordon-Haus effect and because the interaction forces are phase and amplitude sensitive.

In a soliton communication system, the noise power is much smaller than the signal, so we can assume that the noise only produces a small change in the phase and amplitude of each pulse. Making this assumption, we will expect to have a jitter pdf related to the one presented in (12). However, instead of five discrete lines, we should have a five-lobe Gaussian function, with the center of each Gaussian determined by

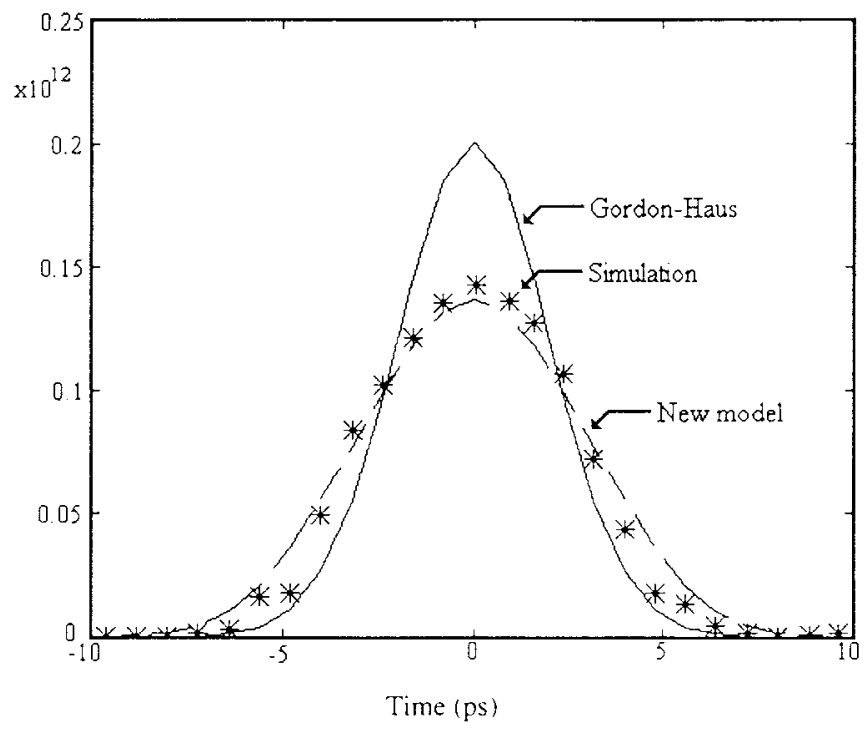

(a)

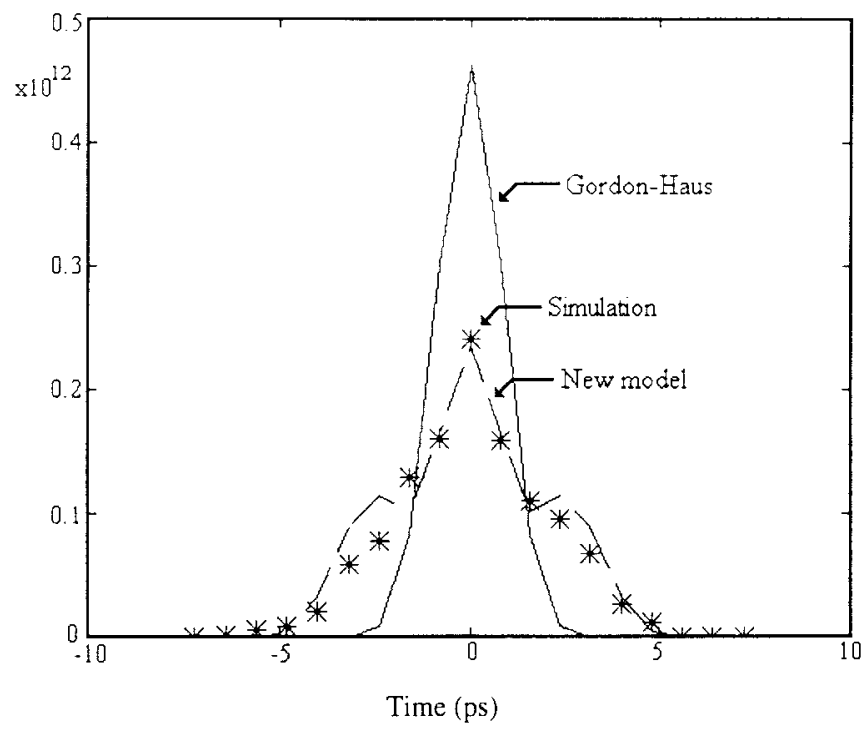

(b)

Fig. 8. Probability density function for (a) a $10-\mathrm{Gb} / \mathrm{s}$ system and (b) a $40-\mathrm{Gb} / \mathrm{s}$ system. The line is the Gordon-Haus result, the starts are the simulation result, and the dashed-line is our model.

expressions (13) and (14), and with the variance given by the Gordon-Haus expression (1). So, we can expect to have a jitter pdf given by

$$
\begin{aligned}
p(t)= & \frac{1}{2} f_{g}(t, \sigma)+\frac{1}{8} f_{g}\left(t-t_{2}, \sigma\right)+\frac{1}{8} f_{g}\left(t+t_{2}, \sigma\right) \\
& +\frac{1}{8} f_{g}\left(t-t_{3}, \sigma\right)+\frac{1}{8} f_{g}\left(t+t_{3}, \sigma\right)
\end{aligned}
$$

where $f_{g}(t, \sigma)$ is given by

$$
f_{g}(t, \sigma)=\frac{1}{\sqrt{2 \pi} \sigma} \exp \left[-\frac{t^{2}}{2 \sigma^{2}}\right]
$$

the values of $t_{2}$ and $t_{3}$ are given by expressions (13) and (14), respectively, and $\sigma$ is given by (1). 


\section{COMPARISON BETWEEN THE ANALYTICAL AND NUMERICAL RESULTS}

To validate our model, we apply it firstly to the simulation results presented in Fig. 1.

As we can see in Fig. 7, the new model gives a good approximation to the pdf of the arrival time. In particular, the tails of the pdf are very well approximated by our model. Since the tails are the main feature determining error rates values in high quality systems, we can expect improved bit error rates estimation applying this model to practical communication systems.

In order to analyze the behavior of the new model for different bit rates we applied it to systems working at 10 and $40 \mathrm{~Gb} / \mathrm{s}$. These are the bit rates of the next generation of optical communication systems. The results are printed in Fig. 8 where we can see again good agreement between the new analytical model and simulation results. Furthermore the deviation of the jitter pdf from the Gaussian shape, is stronger as the bit rate increases.

\section{CONCLUSIONS}

We have derived a simple analytical expression for the jitter pdf of soliton systems, taking into account both soliton interaction and amplified spontaneous emission noise. Monte Carlo simulation results have shown good agreement with analytical ones, thus confirming the validity and accuracy of the new model presented. The soliton interaction produces a considerable deviation from jitter Gaussian statistics for high speed systems.

\section{REFERENCES}

[1] J. P. Gordon and H. A. Haus, "Random walk of coherently amplified solitons in optical fiber transmission," Opt. Lett., vol. 11, no. 10, pp. 665-667, 1986

[2] L. F. Mollenauer, P. V. Mamyshev, and M. J. Neubelt, "Measurement of timing jitter in filter-guided soliton transmission at $10 \mathrm{Gbits} / \mathrm{s}$ and achievement of 375 Gbits/s-Mm, error free, at 12.5 and 15 Gbits/s," Opt. Lett., vol. 19, no. 10, pp. 704-706, 1994.

[3] C. R. Menyuk, "Non-Gaussian corrections to the Gordon-Haus distribution resulting from soliton interactions," Opt. Lett., vol. 20, no. 3, pp. 285-287, 1995.

[4] T. Georges, "Bit error rate degradation of interacting solitons owing to non-Gaussian statistics," Electron. Lett., vol. 31, no. 14, pp. 1174-1175, 1995.

[5] R. F. S. Ribeiro and Jose R. Ferreira da Rocha, "Computationally efficient methods for bit-error rate evaluation in weakly coherent optical systems," J. Lightwave Technol., vol. 12, pp. 1842-1848, Oct. 1994.

[6] J. R. F. da Rocha, L. B. Ribeiro, and A. N. Pinto, "Semi-analytical method for performance analysis of soliton systems," in Proc. CLEO/Pacific Rim'95, Japan, 1995, pp. 43-44.

[7] D. Marcuse, "Simulations to demonstrate reduction of the Gordon-Haus effect," Opt. Lett., vol. 17, no. 1, pp. 34-36, 1992.

[8] V. I. Karpman, and V. V. Solov'ev, " A perturbational approach to the two-soliton systems," Physica 3D, 1981, pp. 487-502.

[9] J. P. Gordon, "Interaction forces among solitons in optical fibers," Opt. Lett., vol. 8, no. 11, pp. 596-598, 1983

[10] M. C. Jeruchim, P. Balaban, and K. S. Shanmugan, Simulation of Communication Systems. New York: Plenum, 1992, pp. 284-287.
[11] G. P. Agrawal, Fiber-Optic Communication Systems. New York: Wiley, 1992, pp. 329-383.

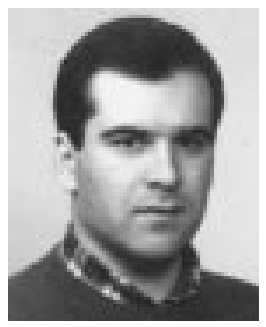

Armando Nolasco Pinto was born in Oliveira do Bairro, Portugal, in 1971. He received the electrical and telecommunications engineering degree from the University of Aveiro, Portugal, in 1994. He is currently pursuing the Ph.D. degree with the Optical Communication Group in the Department of Electronic and Telecommunications, University of Aveiro, Portugal.

His main research interests is in optical soliton communication systems. He has been working in the European soliton communication project called ESTHER in the framework of ACTS (Advanced Communications Technologies and Services).

Mr. Pinto is a student member of the Optical Society of America (OSA).

Govind P. Agrawal (M'83-SM'86-F'96) received the B.S. degree from the University of Lucknow, India, in 1969 and the M.S. and Ph.D. degrees from the Indian Institute of Technology, New Delhi, in 1971 and 1974 respectively.

After holding positions at the Ecole Polytechnique, France, the City University of New York, New York, and AT\&T Bell Laboratories, Murray Hill, NJ, he joined the Faculty of the Institute of Optics at the University of Rochester, Rochester, NY, in 1989, where he is a Professor of Optics. His research interests focus on quantum electronics, nonlinear optics, and laser physics. In particular, he has contributed significantly to the fields of semiconductor lasers, nonlinear fiber optics, and optical communications. $\mathrm{He}$ is an author or coauthor of more than 250 research papers, several book chapters and review articles, and three books entitled Semiconductor Lasers (New York: Van Nostrand Reinhold, 1993), Nonlinear Fiber Optics (New York: Academic, 1995), and Fiber-Optic Communication Systems (New York: Wiley, 1997). He has also edited the books Contemporary Nonlinear Optics (New York: Academic, 1992) and Semiconductor Lasers: Past, Present and Future (New York: AIP Press, 1995).

Dr. Agrawal is a Fellow of the Optical Society of America (OSA).

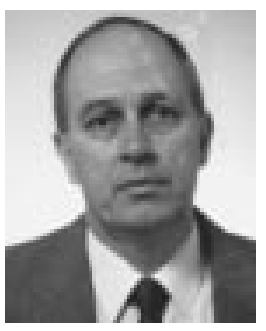

J. Ferreira da Rocha received the degree of "Licenciatura" in electrical engineering from the University of Lourenço Marques (now Eduardo Mondlane), Mozambique, in 1973, the M.Sc. degree in telecommunication systems, and the Ph.D. in electrical engineering from the University of Essex, U.K. in 1980 and 1983 , respectively.

From 1979 to 1983 , he carried out postgraduate research at the University of Essex, U.K., in the area of optical communications receiver optimization. In 1983, he returned back to the University of Aveiro, Portugal, where he is at present a Full Professor and coordinates the University of Aveiro participation in four projects, in the context of the European Union ACTS Program (R\&D in the telecommunications area). He has published some 130 papers mainly in international journals and conferences, and his present research interests include modulation formats and receiver design for very high-capacity optical communication systems based on linear and nonlinear (soliton) transmission, applications of low-noise quantum devices to communication systems, and optical networks for mobile radio applications. In the past few years he has acted as a technical auditor and evaluator for projects included in European Union R\&D Programs.

Dr. da Rocha is also a member of COST Technical Committee for Telecommunications (COST, European Program for Scientific and Technical Cooperation). 